Rev. Biol. Trop., 45(4): 1421-1428, 1997

\title{
Variación diurna de la materia orgánica particulada en una laguna costera del Golfo de California
}

\author{
A. Martínez López e I.Gárate Lizárraga. \\ Laboratorio de Fitoplancton, Departamento de Plancton y Ecología Marina, Centro Interdisciplinario de Ciencias Marinas, \\ CICIMAR-IPN Apartado Postal 592 La Paz, Baja California Sur, México, C.P. 23000.
}

Recibido 4-IV-1996. Corregido 13-Xi-1996. Aceptado 1-VIII-1997.

\begin{abstract}
Three diel cycles of sampling were carried out in February, May, and October 1991 at the mouth of Bahía Concepción. This was done to describe the diurnal variability of size-fractionated phytoplankton abundance, species composition, pigments, and particulate organic matter (POM) in two depths. These variables show a very marked monthly variation, with February being the richest period in organic particles. Comparing the results obtained in the three cycles, we can observe that no significant differences $(\mathrm{p}=0.05 \%)$ exist for both sampling levels during February and May. However, temperature, abundance, and species composition show significant differences $(\mathrm{p}=0.05 \%)$ between sampling levels during October. On the other hand, our results show a correlation between the import and export of organic matter particles during the tide phases. The analysis of variance shows significant differences $(95 \%$ confidence) in the POM concentrations in the February and October months. In February, the highest concentrations were recorded during the high tide. In October, an inverse condition was observed. The results of these cycles display the influence of the Gulf of California waters flowing into Bahía Concepción in February bringing in particulate organic matter. During October, an outflow of particles from the bay to the ocean was observed. In May an intermedian condition was observed.
\end{abstract}

Key words: Diel variation, particulate organic matter, phytoplankton, Concepcion Bay, Gulf of California.

Las lagunas costeras se ubican dentro de los ecosistemas naturales de más alta productividad primaria y secundaria. Esta productividad se debe a numerosos subsidios de energía y a las diferentes actividades de los productores primarios y secundarios (Yañez 1986). En algunos cuerpos costeros, la intensidad de las corrientes de mareas son el principal factor ecológico, ya que influye en los patrones de circulación y determina la intensidad temporal de las interacciones químicas y biológicas en ciclos semidiurnos y diurnos (Alvarez et al. 1975). Por ello, los procesos biológicos al interior de estos cuerpos de agua, adquieren una mayor dinámica regulada principalmente por los ciclos de marea.

Debido a la naturaleza abierta de este tipo de sistemas, es característico un transporte pasivo de sedimentos suspendidos, nutrientes orgánicos e inorgánicos y organismos planctónicos, durante los ciclos de marea. Aunque todo el transporte neto del agua es en general hacia al mar, existe movimiento de materiales en ambas direcciones, dependiendo de las condiciones climático-meteorológicas, geomorfológicas e hidrográficas del lugar (Yañez 1986, Farfán y Alvarez 1983). Por lo anterior, el objetivo del presente trabajo es describir la variabilidad en la concentraión de partículas orgánicas por efecto de las corrientes de marea durante tres ciclos diurnos realizados en la boca de Bahía Concepción.

\section{MATERIALES Y MÉTODOS}

Bahía Concepción se localiza en el Golfo de California entre los $26^{\circ} 33^{\prime}$ y $26^{\circ} 53^{\prime} \mathrm{N}$ y 
$111^{\circ} 42^{\prime}$ y $112^{\circ} 56^{\prime} \mathrm{W}$. Esta bahía presenta una marea de tipo mixto semiduirno (Obeso et al. 1996). En febrero, mayo y octubre de 1991 se tomaron muestras de agua para el análisis de clorofila a y b, proteínas, carbohidratos, abundancia fitoplanctónica y temperatura en muestreos diumos en una estación ubicada en la boca de Bahía Concepción. De manera simultánea, se midió la marea mediante una regla de marea. Para la cuantificación del nanofitoplancton (organismos $<20 \mu \mathrm{m}$ ) y del microfitoplancton (organismos $>20 \mu \mathrm{m}$ ), se tomaron 250 $\mathrm{ml}$ de agua y se fijaron con formol neutralizado con borato de sodio al $4 \%$. El análisis de las muestras se realizó por el método de Utermöhl (Hasle 1978). Para la determinación de la concentración de pigmentos fotosintéticos, carbohidratos y proteínas se filtraron $500 \mathrm{ml}$ de agua en filtros tipo Whatman GF/F, análizándose posteriormente por los métodos espectrofotométricos de Jeffrey y Humphrey (1975), Dubois et al. (1956) y Lowry et al. (1951), respectivamente. La contribución del fitoplancton (CF) y la del material particulado no fotosintético no fitoplanctónico $(\mathrm{CNF})$ a la biomasa total estimada por proteínas y carbohidratos fueron calculados de acuerdo con lo propuesto por Lancelot (1980). Se realizó un análisis de diferencia de medias para comparar si había diferencias significativas entre ambos niveles de muestreo (Downie y Heath 1986) y un análisis de varianza para cada ciclo diurno para probar las diferencias en la concentración del MOP durante el flujo y reflujo de la marea.

\section{RESULTADOS}

Se observaron cambios importantes en la variación de la temperatura de un muestreo a otro. Febrero fue el más frío, con un valor promedio para ambos niveles de $17.7^{\circ} \mathrm{C}$. Mayo presentó valores promedio de $22.6^{\circ} \mathrm{C}$ y octubre fue el mes más caliente con un promedio de $28^{\circ} \mathrm{C}$ (Cuadro 1). La tendencia de los datos reflejó el calentamiento durante el ciclo diurno. En cuanto a la temperatura entre superficie y $10 \mathrm{~m}$, esta solo fue significativamente $(p=0.05 \%)$ diferente en octubre.

Para los tres ciclos diurnos se identificaron un total de 124 taxa, de los cuales 80 fueron diatomeas, 36 dinoflagelados, 6 silicoflagelados, 1 cianofita y 1 euglenofita. El mayor número de especies correspondió al mes de febrero, después al de octubre y por último al de mayo. La comunidad fitoplanctónica en febrero estuvo representada por Chaetoceros curvisetus, Ch. debilis y $\mathrm{Ch}$. costatus, en mayo estuvo compuesta por Rhizosolenia imbricata, Rh. stolterfothii y Chaetoceros spp. En octubre los taxa dominantes fueron Eucampia zodiacus, E. cornuta, Oscillatoria sp, $N$. pacifica y Pseudonitzschia pseudodelicatissima. Comparando las especies presentes en ambos niveles de muestreo, se observó que existen diferencias en cuanto a las especies dominantes solo en octubre (Fig. 1).

\section{CUADRO 1}

Valores promedio de temperatura, marea, clorofilas a y $b$, intervalo de valores de la relación clorofila b:a, carbohidratos y proteínas particulados, y abundancia fitoplanctónica fraccionada (micro y nanofitoplancton) en febrero, mayo y octubre de 1991.

\begin{tabular}{|c|c|c|c|c|c|c|c|c|c|c|}
\hline Mes & Prof. (m) & $\mathrm{T}\left({ }^{\circ} \mathrm{C}\right)$ & $\begin{array}{c}\text { Marea } \\
(\mathrm{cm})\end{array}$ & $\begin{array}{l}\mathrm{Chl} \mathrm{a} \\
\mathrm{mg} / \mathrm{m} 3\end{array}$ & $\begin{array}{l}\mathrm{Chl} \mathrm{b} \\
\mathrm{mg} / \mathrm{m} 3\end{array}$ & Chl b:a & Cho $\mathrm{mg} / \mathrm{m} 3$ & Prot $\mathrm{mg} / \mathrm{m} 3$ & Micro & Nano \\
\hline \multirow{4}{*}{ Febrero } & 0 & 17.8 & \multirow{4}{*}{60} & 1.91 & 0.03 & $0.06-0.38$ & 140 & 307 & 400 & 269 \\
\hline & & & & & & & & & & \\
\hline & 10 & 17.7 & & 2.53 & 0.09 & $0.06-0.14$ & 134 & 292 & 364 & 307 \\
\hline & 0 & 22.5 & & 0.06 & 1.13 & $0.32-2.75$ & 151 & 49 & 34 & 261 \\
\hline \multirow[t]{3}{*}{ Mayo } & & & \multirow[t]{3}{*}{127} & & & & & & & \\
\hline & 10 & 22.6 & & 0.41 & 0.73 & $0.22-3.1$ & 160 & 87. & 45 & 295 \\
\hline & 0 & 28.2 & & 0.07 & 0.01 & $0.11-0.46$ & 115 & 50 & 67 & 34 \\
\hline \multirow[t]{2}{*}{ Octubre } & & & \multirow[t]{2}{*}{90} & & & & & & & \\
\hline & 10 & 27.7 & & 0.90 & 0.09 & $0.13-1.15$ & 146 & 69 & 31 & 325 \\
\hline
\end{tabular}



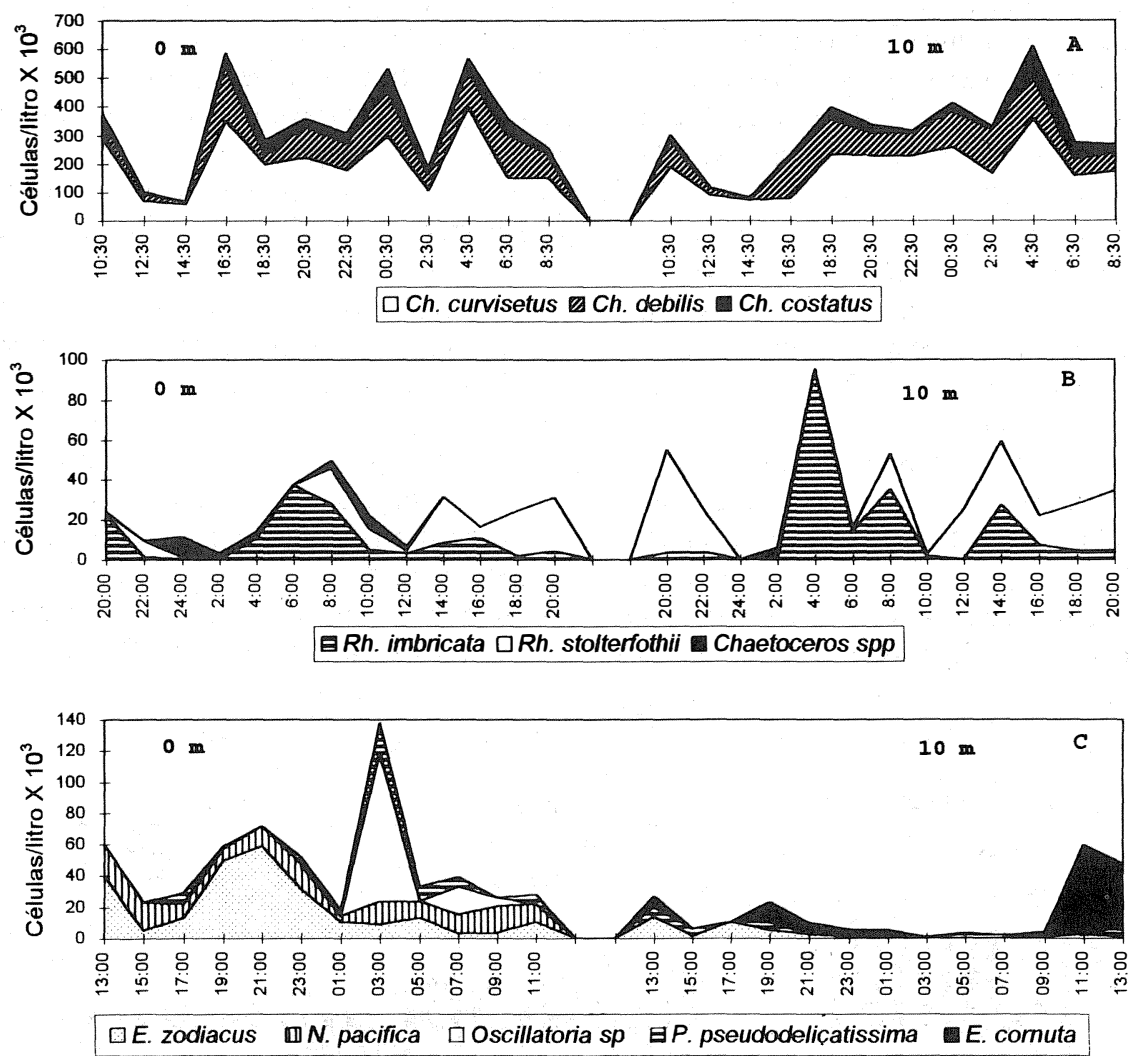

Figura 1.- Variación diurna de las especies más abundantes en ambos niveles de muestreo para los ciclos de febrero (a), mayo (b) y octubre (c) de 1991.

En superficie se observó que la abundancia fitoplanctónica total disminuyó de un ciclo a otro, presentando los valores promedios más altos en febrero $\left(652 \times 10^{3} \mathrm{cel} / \mathrm{l}\right)$, intermedios en mayo $\left(249 \times 10^{3} \mathrm{cel} / \mathrm{l}\right)$ y menores en octubre (101 $\mathrm{x} 10^{3} \mathrm{cel} / \mathrm{l}$ ) (Fig.2, Cuadro 1). A 10 metros, la reducción en la abundancia fue más clara entre febrero y mayo (Fig. 3). En febrero se observó que el microfitoplancton dominó sobre el nanofitoplancton en el $83 \%$ de las muestras, mientras que en mayo el nanofitoplancton dominó en el $100 \%$ de las muestras en ambos niveles (Figs. 2 y 3; Cuadro 1). En octubre la fracción dominante en superficie fue el microfitoplancton, mientras que a $10 \mathrm{~m}$ se observó un dominio total de las células pequeñas (Figs. 2 y 3; Cuadro
1).La variación de la concentración media de la materia orgánica particulada (MOP) y la clorofila $a$, muestra que las mayores concentraciones tanto de proteínas $\left(292-306 \mathrm{mg} / \mathrm{m}^{3}\right)$, como de clorofila $\boldsymbol{a}\left(1.91-2.53 \mathrm{mg} / \mathrm{m}^{3}\right)$ se presentaron en el mes de febrero, disminuyendo en forma acusada en los dos meses siguientes. En cuanto a los carbohidratos, no se observaron cambios importantes en las concentraciones entre un ciclo y otro, aunque los mayores valores $\left(151-160 \mathrm{mg} / \mathrm{m}^{3}\right)$ se obtuvieron en mayo, (Fig. 2 y 3; Cuadro 1).El análisis de varianza reveló diferencias significativas por arriba del $95 \%$, entre las fases de la marea en las concentraciones de MOP en febrero y octubre. En febrero, la marea alta correspondió con las mayores concentraciones de partículas, mientras que en octubre se presentó una situación 
inversa. En este último mes, se encontró una mayor cantidad de proteínas y carbohidratos a 10 $\mathrm{m}$, siendo los carbohidratos los principales contribuyentes a la MOP. Asimismo, en superficie se observaron las mayores concentraciones de ambas especies químicas con la salida de la marea, así como la disminución de las concentraciones de ésta, a medida que se invierte la dirección del flujo de marea (Figs. 2 y 3). Las contribuciones del fitoplancton (CF) y del material particulado no fitoplanctónico (CNF) a la biomasa total en superficie, mostraron un claro descenso de la CF a las proteínas de febrero a octubre, en el agua que entra en la bahía. Por el contrario, en el agua que sale de la bahía, esta fracción incrementa considerablemente su porcentaje de participación en octubre (Fig. 4A). Este mismo patrón se conserva a $10 \mathrm{~m}$ aunque no tan claramente (Fig. 4B).Es importante señalar la escasa contribución del fitoplancton a los carbohidratos, sobretodo en superficie durante febrero y en ambos niveles en mayo, esto significa que en estos meses los carbohidratos del material detrítico o no fitoplanctónico en el agua que sale de la bahía se incrementó a porcentajes mayores del $90 \%$ (Fig. 5A y B). Un hecho que resulta interesante en relación al fitoplancton, es el haber encontrado altas concentraciones de clorofila b, particularmente en mayo, lo cual trajo como consecuencia que los coeficientes clorofila $b: a$ se incrementaran notablemente en este mes y en ambos niveles de muestreo (Cuadro 1): En octubre este incremento solo se observó a $10 \mathrm{~m}$ de profundidad.
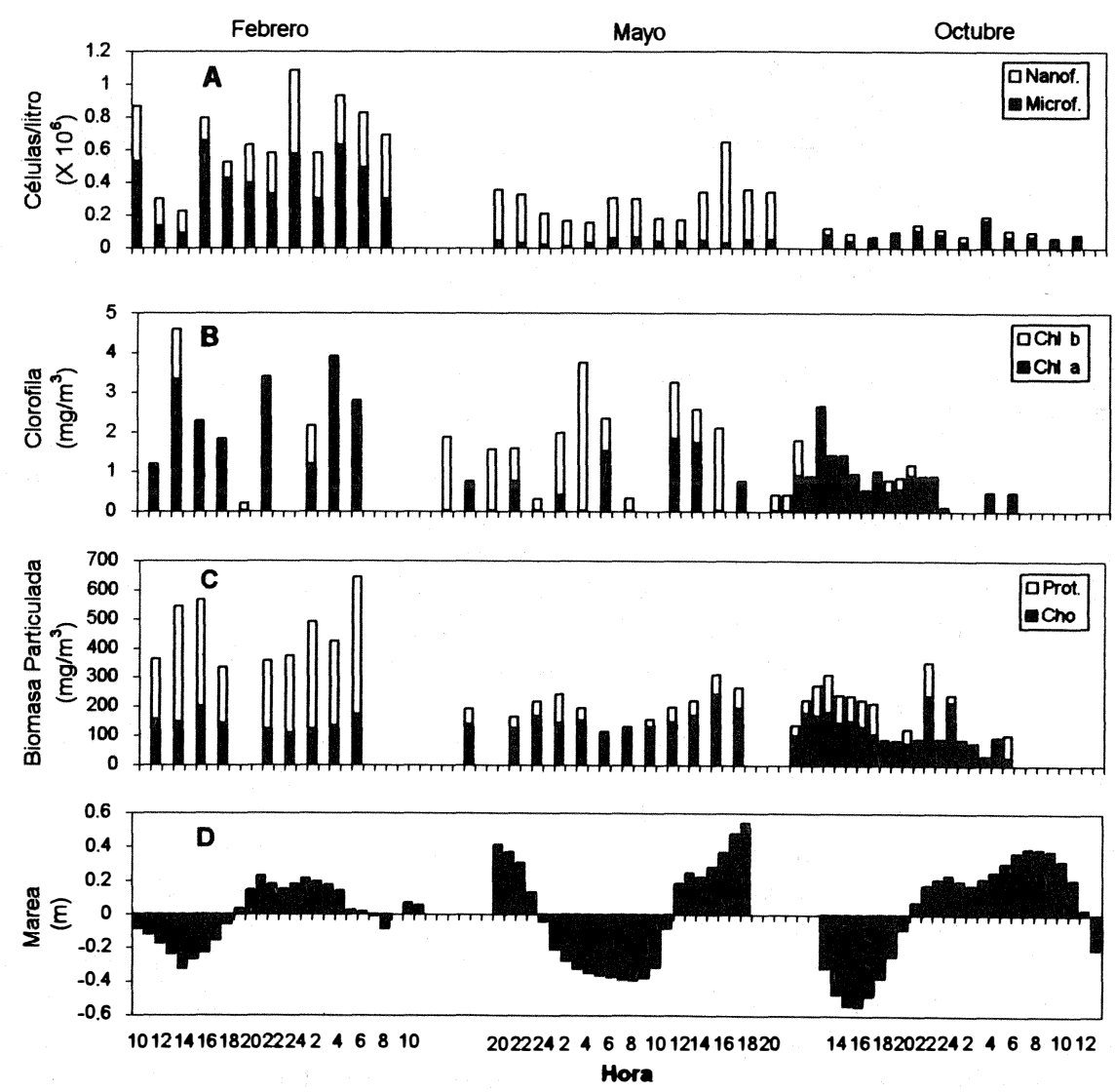

Figura 2.- Variación diurna de la abundancia fitoplanctónica total (a); pigmentos (b), biomasa orgánica particulada (c) en superficie, en los muestreos de febrero (a), mayo (b) y octubre (c) de 1991. 

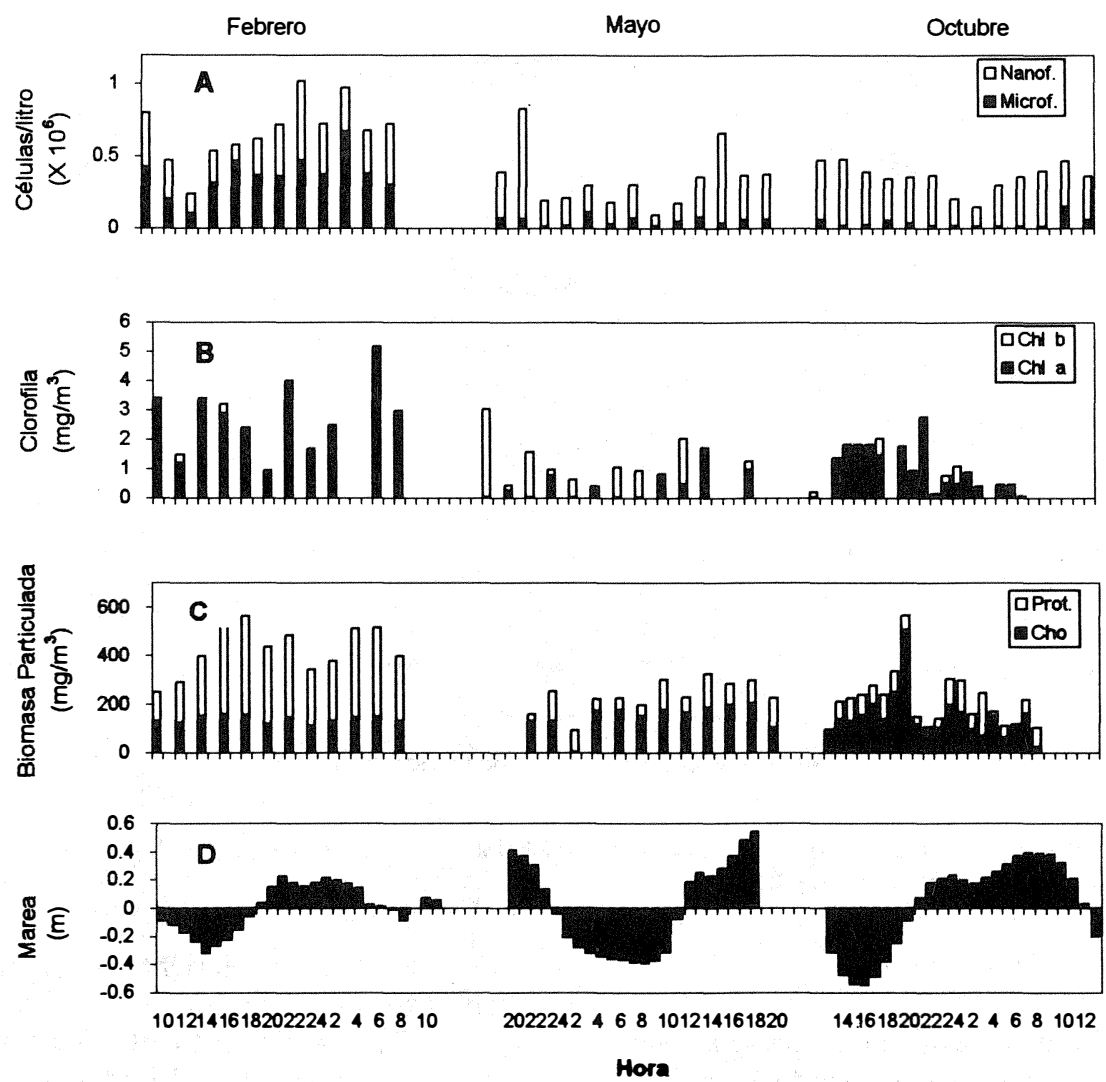

Figura 3.- Variación diurna de la abundancia fitoplanctónica total (a); pigmentos (b), biomasa organica particulada (c) a 10 m de profundidad en los muestreos de febrero (a), mayo (b) y octubre (c) de 1991.

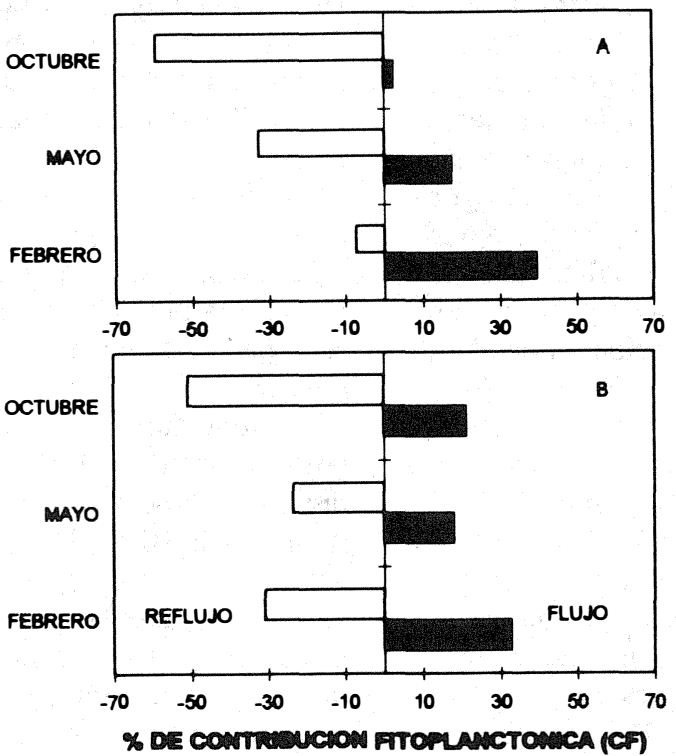

Figura 4.- Porcentaje de contribución del fitoplancton a las proteínas particuladas durante el flujo y reflujo de la marea en febrero, mayo y octubre: A) superficie y B) $10 \mathrm{~m}$. 

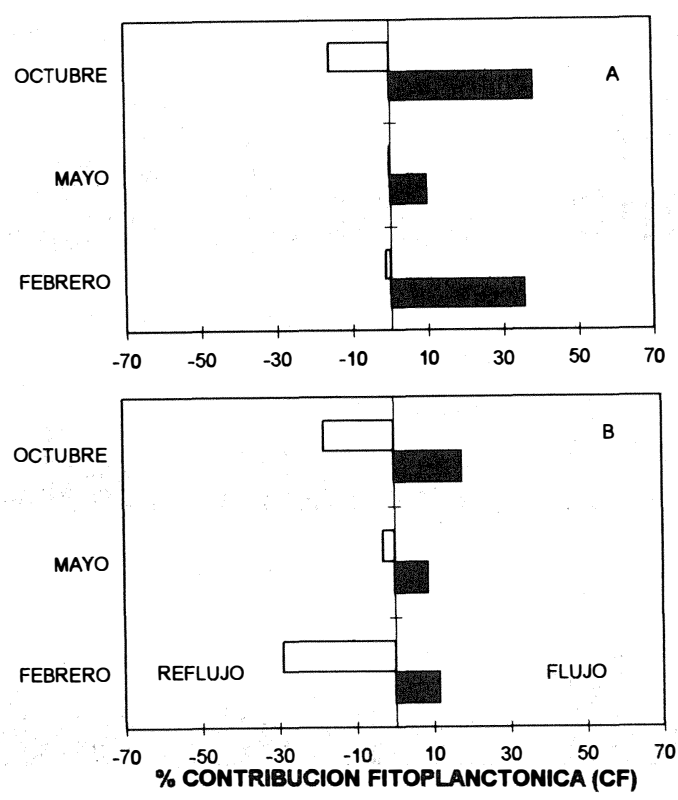

Figura 5.- Porcentaje de contribución del fitoplancton a las carbohidratos particulados durante el flujo y reflujo de la marea en febrero, mayo y octubre: A) superficie y B) $10 \mathrm{~m}$.

\section{DISCUSION}

La variación de la temperatura del agua cambió para las tres muestreos, siendo febrero el periodo más frío y octubre el más caliente, lo cual refleja la marcha del ciclo anual en el Golfo de California, en donde las mayores temperaturas corresponden a los meses de septiembre y octubre (Alvarez y Lara 1991). Por otra parte, la variación diurna de temperatura reflejó el efecto de calentamiento solar, el cual enmascara las diferencias que pudieran existir entre el agua oceánica y la de la bahía. Otro aspecto físico que se puede observar en nuestros resultados, es la homogeneidad entre superficie y $10 \mathrm{~m}$ en febrero y mayo y una estratificación en octubre, coincidiendo con lo reportado por Reyes (1994).

La composición específica del microfitoplancton mostró cambios en cuanto a los taxa dominantes. El microfitoplancton en el ciclo de febrero y en particular de tres especies del género Chaetoceros, reflejan la entrada de agua del golfo, ya que las máximas densidades se registraron con la entrada de la marea, además de que existen reportes de su presencia y abundancia en las aguas aledañas a la bahía durante la temporada invierno-primavera (Cupp y Allen 1938, Cupp 1943). Estos resultados sugieren que existió aporte de nutrientes por mezcla de la columna de agua, provocada por los vientos del noroeste, característicos de esta temporada (Alvarez y Lara 1991), lo cual favoreció la proliferación de este género, típico del primer estadio de la sucesión fitoplanctónica (Margalef 1958). En mayo, el tipo de diatomeas dominantes (Rhizosolenia spp) puede estar sugiriendo una etapa de transición entre condiciones de inestabilidad y enriquecimiento y condiciones de estratificación y oligotrofía al interior de la bahía. En octubre, el intercambio de agua entre la bahía y la zona oceánica adyacente se hace evidente no sólo en la abundancia fitoplanctónica, sino también en la distribución vertical de las especies dominantes. Así, la presencia de $N$. pacifica y Oscillatoria sp, únicamente en el nivel superficial, muestran la salida de estas poblaciones del interior de la bahía. Mientras que las mayores concentraciones de nanofitoplancton a $10 \mathrm{~m}$ de profundidad señalan la entrada de agua del golfo.

$\mathrm{Al}$ comparar las concentraciones medias de proteínas y carbohidratos de este trabajo con las reportadas por Lechuga et al. (1989) y Bustillos (1990) para el Golfo de California, es evidente que los valores de ambas especies químicas son mayores en esta bahía, lo cual 
puede ser una consecuencia del aporte de material no fitoplanctónico a la biomasa total. En febrero, las mayores concentraciones de proteínas, carbohidratos y fitoplancton, detectadas con la entrada de la marea fueron probablemente el resultado de haber muestreado un parche de plancton que estaba siendo importado hacia la bahía, el cual también fue observado durante la bajamar, aunque con diferencias cuantitativas. Estas diferencias, son posiblemente originadas por la retención de partículas debido a la circulación en el interior de la bahía (Martínez y Gárate 1994). La presencia de este parche, igualmente se corrobora con la composición específica, ya que por un lado, las mismas especies se registraron tanto en las condiciones de flujo como de reflujo en ambos niveles de muestreo, y por el otro, se presentaron diferencias con respecto a las poblaciones fitoplanctónicas encontradas hacia el interior (Martínez y Gárate 1994). En este mes en particular el porcentaje alto de proteínas de origen no fitoplanctónico puede deberse a organismos del microzooplancton (larvas de moluscos y copepodos de talla pequeña) que formaron parte de la muestra total. Estos resultados concuerdan con los de Farfán y Alvarez (1983), quienes señalan que dependiendo de la dinámica de la zona adyacente pueden presentarse condiciones de equilibrio, generándose tiempos de residencia altos para los materiales dentro de una laguna. Lo anterior apoya la idea de que en este ciclo existió una importación de carbón orgánico hacia el interior de esta bahía.

Por otra parte, los datos de mayo señalan que no existen diferencias significativas entre la entrada y la salida de partículas a la bahía, debido quizás a que la amplitud de marea fue la más pequeña de los tres ciclos. Sin embargo, dos aspectos importantes fueron observados en este mes: el primero fue una mayor concentración de carbohidratos con respecto a febrero, la cual fue originada por una mayor contribución de material detrítico, como resultado de la degradación de las macroalgas y pastos marinos en el interior de la bahía y el segundo, fueron las inusualmente altas concentraciones de clorofila b. El origen de la misma es difícil atribuirlo a un grupo particular del fitoplancton, sin embargo la presencia de prasinofíceas y euglenofíceas en el nanoplancton, ya reportadas también en trabajos previos como parte importante de la flora de esta bahía, (Gilmartin y Relevante 1978), señalan a estos grupos como una de las posibles fuentes de tal pigmento según lo propuesto por Jeffrey (1976). Además, cabe la posibilidad de que en este cuerpo costero, como en muchos otros de áreas tropicales y subtropicales exista la presencia de procariotes de la clase Prochlorophyta (Goericke y Repeta 1993), los cuales por su reducido tamaño $(0.6-0.8 \mathrm{~mm})$ son difíciles de cuantificar con el método de Utermohl.

Los resultados de octubre sugieren que la degradación del material particulado durante la temporada cálida libera nutrientes, los cuales propician el desarrollo de poblaciones del microfitoplancton, principalmente hacia el interior de la bahía. Esto permite una exportación de partículas de material orgánico y de energía excedente, conforme al patrón general descrito para las lagunas costeras (Contreras 1989). Nuestros resultados en general, muestran una correlación entre la entrada y salida de partículas de material orgánico durante las fases de marea, confirmándose así la interacción en ambos sentidos, entre el golfo de California y este cuerpo de agua. Sin embargo, con estos muestreos no fue posible describir toda la variabilidad a la que están sujetos los componentes de la materia orgánica particulada en Bahía Concepción, ya que están expuestos a una gran inestabilidad de las condiciones físicoquímicas, las cuales fluctúan en periodos cortos de tiempo y son el resultado principal de las corrientes de marea.

\section{AGRADECIMIENTOS}

A la Dirección de Estudios Profesionales y de Investigación del Instituto Politécnico Nacional, por el apoyo económico para la realización del proyecto "Dinámica de Nutrientes y Plancton en Bahía Concepción, B.C.S.", clave DEPI 903371. Los autores son becarios COFAA.

\section{REFERENCIAS}

Alvarez B.S., G. Ballesteros \& A. Chee. 1975. Estudio de algunas variables físico-químicas superficiales en Bahía San Quintín, en verano, otoño e invierno. Ciencias Marinas :16-45. 
Alvarez, B.S. \& J.R. Lara. 1991. The physical environmental and primary productivity of the Gulf of California. In: , B.R.T. Simoneit \& A. Drophin (Eds). The Gulf and Peninsular Province of the Californias. Am. Assoc. Petr. Geol. Mem., 47. 555-565.

Bustillos, G.J.J. 1990. Biomasa proteica, de carbohidratos y clorofilas de las fracciones de nanoparticulas y microparticulas de la región de las grandes islas y central del Golfo de California. Tesis de Maestría, CICIMAR, La Paz, México.

Contreras, E. F. 1989. Las lagunas costeras mexicanas. Centro de Ecodesarrollo, Secretaria de Pesca, México. $121 \mathrm{p}$.

Cupp, E.E. 1943. Marine Plankton diatoms of the west coast of North America. Bull. Scripps Inst. Oceanogr. 5:1-237.

Cupp, E.E. \& W.E. Allen. 1938. Plankton diatoms of the Gulf of California obtained by the Allan Hanckock Pacific Expeditions of 1937. Univ. Southern Calif. 3:91-99.

Downie N.M. \& R.W. Heath. 1986. Métodos estadísticos aplicados. Harla, México, D.F.. 380 p.

Dubois, M.K., A. Guilles, J.K. Hamilton, P.A. Rebers \& U.F. Smith. 1956. Colorimetric methods for determination of sugar and related sustances. Anal. Chem. $28: 350-356$

Farfán, B.C. \& S. A. Borrego. 1983. Variability and fluxes of nitrogen and organic carbon at the mouth of a costal lagoon. Est. Coast.Shelf Sci. 17:599-612.

Gilmartin, M. \& N. Revelante. 1978. The phytoplankton characteristics of the Barrier island lagoons of the Gulf of California. Est. Coastal Mar. Sci. 7:29-47.

Goericke, R. \& D.J. Repeta. 1993. Chlorophylls a and b and devinyl clorophylls $\mathrm{a}$ and $\mathrm{b}$ in the open subtropical North Atlantic Ocean. Mar. Ecol. Prog. Ser.. 101:307313.

Hasle, G.R. 1978. Using the inverted microscope. In: A. Sournia (ed.). Phytoplankton manual. Paris, 191-196 p.
Jeffrey, S.W. \& G.F. Humphrey. 1975. New spectrophotometric equations for determinig cholorophyls a,b,cl and $c 2$ in algal phytoplankton and higher plants. Biochem, Physiol. Pfanz. B.P.P. 1967:191-194.

Jeffrey, S.W. 1976. A report of grenn algal pigments in the Central North Pacific Ocean. Mar. Biol. 37:33-37.

Lancelot, V. B. C. 1980. Statistical method to estimate the chemical composition of phytoplankton in the Southern Bigth of the North Sea. Est. Coast. Mar. Sci. 10:467-478.

Lechuga D.C.H., B.R. Ayala \& I. L. Gárate. 1989. Proteínas, carbohidratos y clorofila $a$ de la materia orgánica particulada, en tres diferentes ambientes oceánicos: Mar de Portugal, Golfo de California y NW de Baja California Sur. An. Inst. Cienc. del Mar y Limnol. Univ. Nal. Autón. México. 16:232-244.

Lowry, O.H., N.J. Rosenbrough, A.L. Farr \& R.J. Randall. 1951. Protein measurment with folin reagent. J. Biol. Chem. 193:265-275.

Margalef, R. 1958. Temporal succesion and spatial heterogeneity in phytoplankton. In: Buzzati-Traverso. A.A. (ed.). Perspectives in Marine Biology. University of California, Berkeley, p.. 323-349.

Martínez, L A. \& I.L. Gárate. 1994. Cantidad y calidad materia orgánica particulada en Bahía Concepción en la temporada de reproducción de la almeja catarina Argopecten circularis (SOWERBY, 1835). Ciencias Marinas 20:301-320.

Obeso, N., M., M.A. Alatorre v A.R. Jiménez. 1996. Modelación de la marea en Bahía concepción, B.C.S. México.Oceánides 11:1-8.

Reyes, S.A. 1994. Relación entre estructura hidrográfica y la abundancia, distribución y origen de diferentes expresiones de biomasa del seston orgánico en Bahía Concepción. Tesis de Licenciatura, Universidad Nacional Autónoma de México, D.F. México.

Yáñez A., A. 1986. Ecología de la zona costera: Análisis de siete tópicos. AGT,. México, D.F., 190 p. 